\title{
Microring Mixers and Tunable Filters in Silicon Photonics (Project Report 1201308-Y3)
}

\section{SHAYAN MOOKHERJEA}

Department of Electrical and Computer Engineering, University of California, San Diego, MC 0407 La Jolla CA 92093-0407 USA

Email:smookher@ucsd.edu

\section{Introduction and Goals}

The main goal of this NSF-funded project [1201308 - Year 3] is to develop integrated photonics devices based on silicon photonics which can be used for compact and efficient nonlinear classical and quantum photonics applications.

"Mixers" in the title reflects the use of nonlinear optical wave mixing, and the word is borrowed from the microwave devices field, where mixers are in wide usage. An example of four-wave mixing (FWM) is shown in Fig. 1. This is the

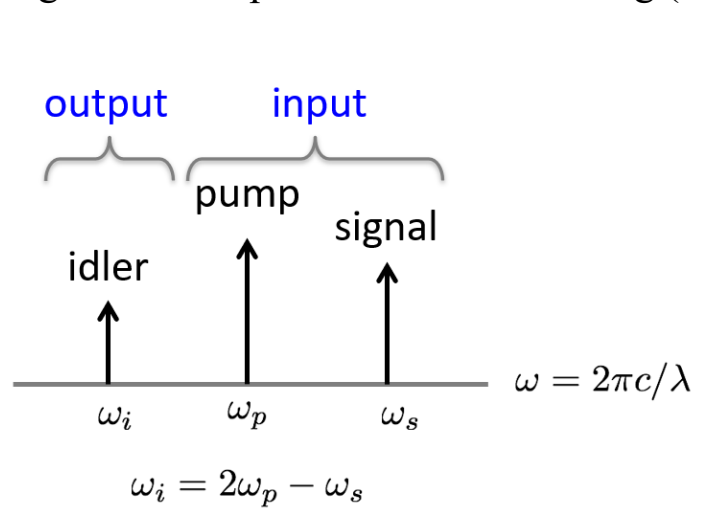

Figure 1 The nonlinear interactions in optical four-wave mixing (FWM) which are used in developing silicon photonics based optical mixers. foundational nonlinear optical process for wide-span wavelength conversion and also for entangled photon-pair generation in silicon photonics, among many other potential applications. Achieving a high conversion efficiency (output idler power divided by input signal power) while mitigating optical propagation losses through the device structure is a central goal of this project. This project studies wavelength conversion in waveguides, microring resonators and coupled-resonator optical waveguides (CROWs) [1], which may be useful for filters [2] and lasers [3], [4], among other types of photonic devices that are typically realized at the microchip scale.

Some degree of fabrication disorder is inevitable in foundry-fabricated silicon photonics. We considered for adoption and implementation a number of ideas such as post-fabrication trimming using high-resolution experimental techniques which we had previously developed [5],[6]. However, it was seen that relatively simple thermal control of the device-under-test was adequate for the nonlinear mixing demonstrations performed in this project. 
Note that an accurate design of the waveguide-resonator coupling is important and often requires time-consuming simulations, since there can be significant deviations from conventional coupled-mode theory in the high-index-contrast regime of silicon photonics [7]. Thus, a significant portion of the research activity in this project addresses the challenges of designing high-index contrast devices accurately and comparing the results of fabrication to the design intention.

\section{Activities}

Previously, we have studied the significant dispersion of directional couplers which are used in several places in our integrated circuits [8]. Another area of potential concern is nonlinearity within the coupler itself, which can sometimes affect the transmission function of resonators, especially racetrack resonators, in way that leads to bistability and oscillatory behavior [9]. We could avoid the bistable regime in the measurements that we performed in this project. To help in stability, we usually observe the optically-pumped microring resonator with an infrared camera to verify that the pump is resonant [10], [11].

The CROW devices are designed using racetrack resonators which consist of both straight and curved waveguide sections. Compared to conventional waveguides, a CROW provides significant resonant enhancement of the conversion efficiency [12], [13], but also offers a relatively wide passband (up to several nanometers). CROWs of up to 235 coupled resonators have been successfully fabricated and measured, and a good agreement was achieved between experiments and theory [14]-[16]. The ab-initio simulation of such devices is not computationally feasible on conventional PC workstations, but a preliminary design can be made using analytical derivations already developed. An analytical treatment of pulse propagation clearly shows the beneficial role that could be played by slow light [17], [18] in enhancing mixing properties.

\section{Progress and Impact}

During the third year of this project (2014-15), we demonstrated the combination of an on-chip ring mixer and a tunable filter. Both stages are constructed using microring resonators. The free-spectral range (FSR) of the mixer and the filter stage rings bears a 2:3 harmonic relationship (mutually prime divisors). The filter attenuates the unused pump and residual signal by $50 \mathrm{~dB}$, and allows the generated idler wavelength to pass through with less than $3 \mathrm{~dB}$ loss [19]. This is a significance advancement because unlike RF mixers (for which single-balanced and double-balanced architectures exist), optical mixers usually show very large levels of pump and residual signal powers at the output, often larger than the newly-generated idler wavelength. 
Conventionally, this requires an off-chip filter to be inserted after the chip, incurring insertion loss and taking up extra space, contrary to the rationale for monolithic integration in silicon photonics. Our objective was to demonstrate the monolithic integration of an efficient wavelength-conversion mixer and a high performance filter (i.e., a nonlinear element and a linear, tunable element) on the same chip.

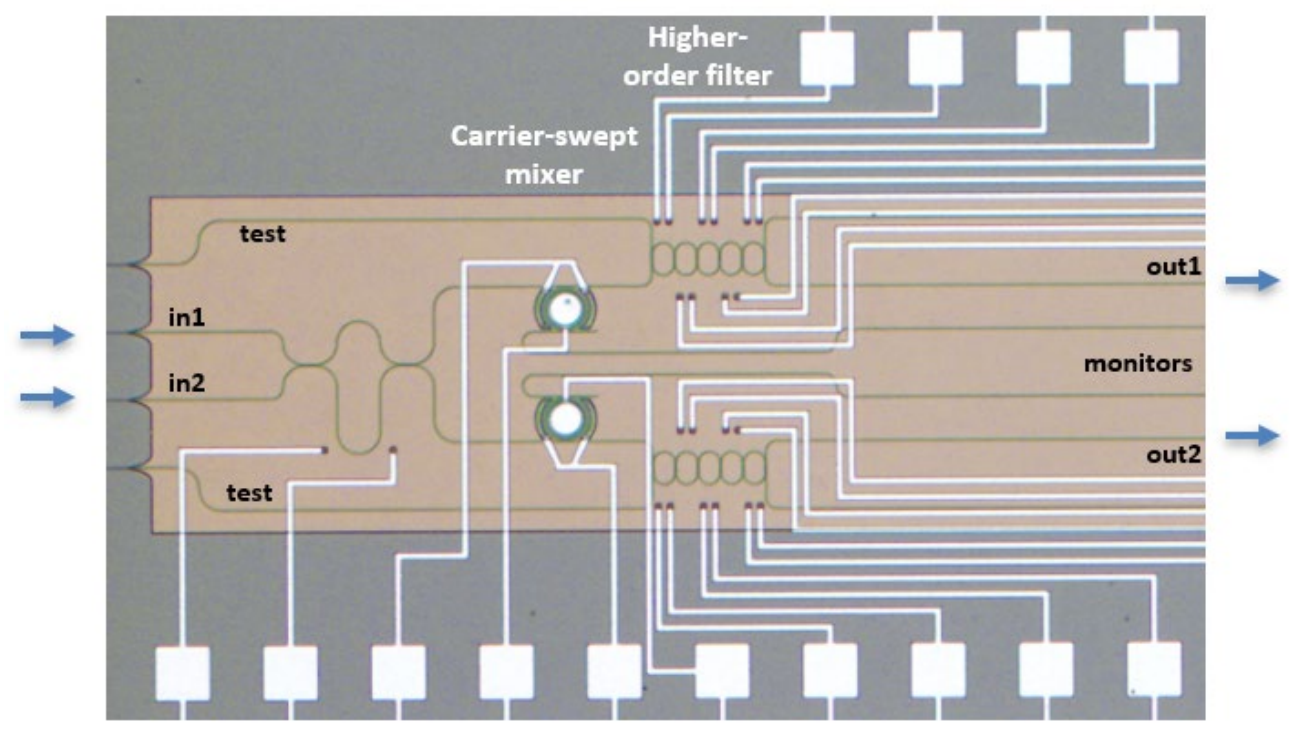

Figure 2 Combination of mixer (four-wave mixing in a silicon microring) and filter (tunable higher-order filter) as part of a silicon photonic integrated circuit. The residual pump and signal attenuated by filter (about $45 \mathrm{~dB}$ ) whereas the generated idler allowed to continue through the filter with less than $3 \mathrm{~dB}$ insertion loss. Two parallel circuits are shown (with inputs labeled In 1 and In2) which operate independently of each other.

Table 1. FWM + Filtering results using the Device shown in Fig. 2

\begin{tabular}{|l|l|l|}
\hline Wavelength & Achieved filtering & Output level (relative to idler) \\
\hline Pump (residual) & $-49.9 \mathrm{~dB}$ & $-10.0 \mathrm{~dB}$ \\
\hline Signal (residual) & $-44.3 \mathrm{~dB}$ & $-21.3 \mathrm{~dB}$ \\
\hline Idler (generated) & $-2.4 \mathrm{~dB}$ & - \\
\hline
\end{tabular}

As Table 1 shows, the residual pump was suppressed in the two-section photonic circuit by a significant amount. Without this level of suppression, the power level in the output port would be completely dominated by the residual pump and the unconverted signal light. After filtering, the dominant component is the newly-converted idler, which comprises about $90 \%$ of the total power.

Because the input pump level was itself high, the final residual pump level was only $10 \mathrm{~dB}$ below that of the idler. Though this is a good first demonstration of a combined mixer + filter functionality in silicon photonics, the level of residual pump suppression is not adequate for use in practical communication systems. 
To ways to improve performance are to seek a further improvement of filtering rejection by about $10 \mathrm{~dB}$, or alternatively, a reduction of the needed pump power to achieve the same level of idler. Ref. [19] proposed a method for using a cascaded high-order CROW filter in order to achieve higher extinction.

We have already studied four-wave mixing experimentally in coupledresonator waveguides [20] and showing improved four-wave mixing in silicon waveguides and in resonators in which a p-i-n diode was fabricated across the waveguide cross-section to sweep out electronic free carriers [21]. Two examples of carrier-swept microring mixers are shown in Fig. 2. Such devices were also used, for example, to realize an time-energy basis entangled photonpair source at room temperature [22].

The concept of all-optical frequency mixing as shown in Fig. 1 can have potential applications in optical communications and all-optical signal processing. Compared to slow thermo-optic tuning [23], all-optical frequency conversion can achieve a much faster response. On the other hand, for wavelength conversion over relatively short frequency ranges (less than 100 $\mathrm{GHz}$ ) can be achieved more simply by using high-bandwidth integrated electrooptic modulators, which were demonstrated in subsequent research [24].

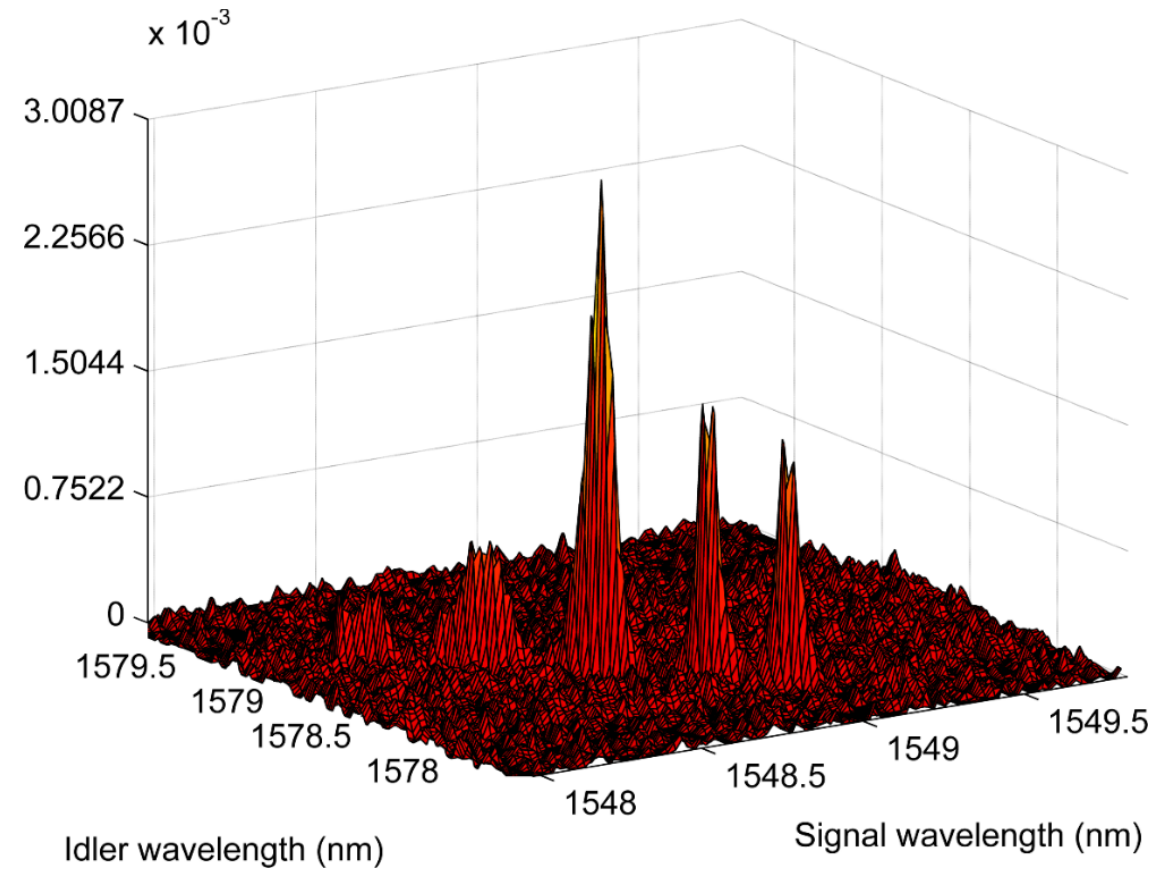

Figure 3 Measurement of a two-photon joint spectral intensity (JSI) for a quantum state of light generated using SFWM in a coupled-resonator optical waveguide (CROW). The Schmidt number $\mathrm{K}=4.01$, indicative of higher-dimensional entanglement generated directly by optical pumping of the device. 
Quantum light generation on an integrated photonic chip is a topic of current interest [25]. Although the second-order chi-(2) nonlinearity of some materials such as lithium niobate [26] is obviously much stronger than the third-order chi-(3) nonlinearity of silicon, it is possible to achieve quite a good level of performance by optimization of the pair-generation properties of silicon microring resonators [27], [28]. Using these insights, we demonstrated the ability to measure and control the generated quantum spectrum of photon pairs. The underlying physical process is that of spontaneous four-wave mixing (SFWM), which is closely related to the mixer concept shown in Fig. 1 (only the pump is input to the device). We showed two methods of controlling the two-photon spectrum (joint spectral intensity, JSI). One is based on finelytuning the pump wavelength and the other is based on tuning the microchip temperature while keeping the pump wavelength fixed. We demonstrated the ability to achieve Schmidt numbers from close to 1 (separable state; suitable for heralded single photons) to almost 10 (high dimensional entanglement). An example of an intermediate state with $\mathrm{K} \sim 4$ is shown in Fig. 3.

Impact on Human Resources: NSF funds partially supported two graduate students and one undergraduate student and one post-doctoral scholar. They worked on device design, fabrication and testing.

\section{Subsequent Extensions}

Because of the unconventional nature of optical propagation [30]-[32], disorder in CROWs can manifest a range of complex physical behavior including localization of light, which can sometimes be controlled electronically [33]. A deeper understanding of the transition between localization and extended propagation regimes allows us to identify which spectral bands are useful for operation under different experimental conditions.

\section{Open-Access Reporting Initiative}

PRAISE: This open-access document is provided in support of our PRAISE

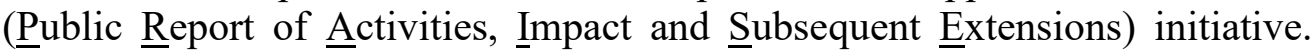
What is it? An open-access document shared with the public which describes the research outcomes of publicly-funded projects. For us, these projects are typically funded by the NSF (National Science Foundation).

\section{References}

[1] F. Morichetti, C. Ferrari, A. Canciamilla, and A. Melloni, "The first decade of coupled resonator optical waveguides: bringing slow light to 
applications," Laser \& Photon. Rev., vol. 6, no. 1, pp. 74-96, Jan. 2012, doi: 10.1002/lpor.201100018.

[2] S. Mookherjea, "Spectral characteristics of coupled resonators," J. Opt. Soc. Am. B, vol. 23, no. 6, p. 1137, Jun. 2006, doi: 10.1364/JOSAB.23.001137.

[3] S. Mookherjea, "Semiconductor coupled-resonator optical waveguide laser," Appl. Phys. Lett., vol. 84, no. 17, pp. 3265-3267, Apr. 2004, doi: 10.1063/1.1719278.

[4] S. Mookherjea, "Using gain to tune the dispersion relation of coupledresonator optical waveguides," IEEE Photon. Technol. Lett., vol. 18, no. 5, pp. 715-717, Mar. 2006, doi: 10.1109/LPT.2006.871144.

[5] Y. Shen, I. B. Divliansky, D. N. Basov, and S. Mookherjea, "Electricfield-driven nano-oxidation trimming of silicon microrings and interferometers," Opt. Lett., vol. 36, no. 14, p. 2668, Jul. 2011, doi: 10.1364/OL.36.002668.

[6] Y. Shen, I. B. Divliansky, D. N. Basov, and S. Mookherjea, "Perfect setand-forget alignment of silicon photonic resonators and interferometers," in Optical Fiber Communication Conference/National Fiber Optic Engineers Conference 2011, Los Angeles, California, 2011, p. PDPC3. doi: 10.1364/OFC.2011.PDPC3.

[7] M. L. Cooper and S. Mookherjea, "Numerically-assisted coupled-mode theory for silicon waveguide couplers and arrayed waveguides," Opt. Express, vol. 17, no. 3, p. 1583, Feb. 2009, doi: 10.1364/OE.17.001583.

[8] R. Aguinaldo, Yiran Shen, and S. Mookherjea, "Large Dispersion of Silicon Directional Couplers Obtained via Wideband Microring Parametric Characterization," IEEE Photon. Technol. Lett., vol. 24, no. 14, pp. 1242-1244, Jul. 2012, doi: 10.1109/LPT.2012.2198639.

[9] S. Mookherjea and M. A. Schneider, "The nonlinear microring add-drop filter," Opt. Express, vol. 16, no. 19, p. 15130, Sep. 2008, doi: 10.1364/OE.16.015130.

[10] M. L. Cooper, G. Gupta, J. S. Park, M. A. Schneider, I. B. Divliansky, and S. Mookherjea, "Quantitative infrared imaging of silicon-on-insulator microring resonators," Opt. Lett., vol. 35, no. 5, p. 784, Mar. 2010, doi: 10.1364/OL.35.000784.

[11] S. Mookherjea and H. R. Grant, "High dynamic range microscope infrared imaging of silicon nanophotonic devices," Opt. Lett., vol. 37, no. 22, p. 4705, Nov. 2012, doi: 10.1364/OL.37.004705.

[12] S. Mookherjea and A. Yariv, "Second-harmonic generation with pulses in a coupled-resonator optical waveguide," Phys. Rev. E, vol. 65, no. 2, p. 026607, Jan. 2002, doi: 10.1103/PhysRevE.65.026607.

[13] S. Mookherjea and A. Yariv, "Kerr-stabilized super-resonant modes in coupled-resonator optical waveguides," Phys. Rev. E, vol. 66, no. 4, p. 046610, Oct. 2002, doi: 10.1103/PhysRevE.66.046610. 
[14] M. L. Cooper et al., "235-ring Coupled-Resonator Optical Waveguides," in Conference on Lasers and Electro-Optics 2010, San Jose, California, 2010, p. CTuHH3. doi: 10.1364/CLEO.2010.CTuHH3.

[15] S. Mookherjea and M. A. Schneider, "Avoiding bandwidth collapse in long chains of coupled optical microresonators," Opt. Lett., vol. 36, no. 23, p. 4557, Dec. 2011, doi: 10.1364/OL.36.004557.

[16] M. L. Cooper and S. Mookherjea, "Modeling of Multiband Transmission in Long Silicon Coupled-Resonator Optical Waveguides," IEEE Photon. Technol. Lett., vol. 23, no. 13, pp. 872-874, Jul. 2011, doi: 10.1109/LPT.2011.2141657.

[17] S. Mookherjea and A. Yariv, "Optical pulse propagation in the tightbinding approximation," Opt. Express, vol. 9, no. 2, p. 91, Jul. 2001, doi: 10.1364/OE.9.000091.

[18] S. Mookherjea and A. Yariv, "Optical pulse propagation and holographic storage in a coupled-resonator optical waveguide," Phys. Rev. $E$, vol. 64, no. 6, p. 066602 , Nov. 2001, doi: 10.1103/PhysRevE.64.066602.

[19] J. R. Ong, R. Kumar, and S. Mookherjea, "Silicon microring-based wavelength converter with integrated pump and signal suppression," Opt. Lett., vol. 39, no. 15, p. 4439, Aug. 2014, doi: 10.1364/OL.39.004439.

[20] J. R. Ong et al., "Low-power continuous-wave four-wave mixing in silicon coupled-resonator optical waveguides," Opt. Lett., vol. 36, no. 15, pp. 2964-2966, 2011.

[21] J. R. Ong, R. Kumar, R. Aguinaldo, and S. Mookherjea, "Efficient CW Four-Wave Mixing in Silicon-on-Insulator Micro-Rings With Active Carrier Removal," IEEE Photon. Technol. Lett., vol. 25, no. 17, pp. 1699 1702, Sep. 2013, doi: 10.1109/LPT.2013.2272521.

[22] M. Savanier, R. Kumar, and S. Mookherjea, "Optimizing photon-pair generation electronically using a $p-i-n$ diode incorporated in a silicon microring resonator," Appl. Phys. Lett., vol. 107, no. 13, p. 131101, Sep. 2015, doi: 10.1063/1.4932047.

[23] R. Aguinaldo et al., "Wideband silicon-photonic thermo-optic switch in a wavelength-division multiplexed ring network," Opt. Express, vol. 22, no. 7, p. 8205, Apr. 2014, doi: 10.1364/OE.22.008205.

[24] X. Wang, P. O. Weigel, J. Zhao, M. Ruesing, and S. Mookherjea, "Achieving beyond-100-GHz large-signal modulation bandwidth in hybrid silicon photonics Mach Zehnder modulators using thin film lithium niobate," APL Photonics, vol. 4, no. 9, p. 096101, Sep. 2019, doi: 10.1063/1.5115243.

[25] J. R. Ong and S. Mookherjea, "Quantum light generation on a silicon chip using waveguides and resonators," Opt. Express, vol. 21, no. 4, p. 5171, Feb. 2013, doi: 10.1364/OE.21.005171. 
[26] J. Zhao, C. Ma, M. Rüsing, and S. Mookherjea, "High Quality Entangled Photon Pair Generation in Periodically Poled Thin-Film Lithium Niobate Waveguides," Phys. Rev. Lett., vol. 124, no. 16, p. 163603, Apr. 2020, doi: 10.1103/PhysRevLett.124.163603.

[27] C. Ma, X. Wang, V. Anant, A. D. Beyer, M. D. Shaw, and S. Mookherjea, "Silicon photonic entangled photon-pair and heralded single photon generation with CAR $>12,000$ and $\mathrm{g}^{\wedge}(2)(0)<0006$," Opt. Express, vol. 25, no. 26, p. 32995, Dec. 2017, doi: 10.1364/OE.25.032995.

[28] R. Kumar, M. Savanier, J. R. Ong, and S. Mookherjea, "Entanglement measurement of a coupled silicon microring photon pair source," Opt.

Express, vol. 23, no. 15, p. 19318, Jul. 2015, doi: 10.1364/OE.23.019318.

[29] X. Wang et al., "Wide-range and fast thermally-tunable silicon photonic microring resonators using the junction field effect," Opt. Express, vol. 24, no. 20, p. 23081, Oct. 2016, doi: 10.1364/OE.24.023081.

[30] S. Mookherjea and A. Yariv, "Pulse propagation in a coupled resonator optical waveguide to all orders of dispersion," Phys. Rev. E, vol. 65, no. 5, p. 056601, Apr. 2002, doi: 10.1103/PhysRevE.65.056601.

[31] M. L. Cooper et al., "Waveguide dispersion effects in silicon-oninsulator coupled-resonator optical waveguides," Opt. Lett., vol. 35, no. 18, p. 3030, Sep. 2010, doi: 10.1364/OL.35.003030.

[32] S. Mookherjea, D. S. Cohen, and A. Yariv, "Nonlinear dispersion in a coupled-resonator optical waveguide," Opt. Lett., vol. 27, no. 11, p. 933, Jun. 2002, doi: 10.1364/OL.27.000933.

[33] S. Mookherjea, J. R. Ong, X. Luo, and L. Guo-Qiang, "Electronic control of optical Anderson localization modes," Nature Nanotech, vol. 9, no. 5, pp. 365-371, May 2014, doi: 10.1038/nnano.2014.53. 